\title{
The Effectiveness of Traditional Chinese Medicine in Treating Patients with Leukemia
}

\author{
Yu-Jun Wang, ${ }^{1}$ Chung-Chih Liao, ${ }^{2}$ Hsuan-Ju Chen, ${ }^{3,4}$ \\ Ching-Liang Hsieh, ${ }^{1,5}$ and Tsai-Chung $\mathrm{Li}^{6,7,8}$ \\ ${ }^{1}$ Graduate Institute of Integrated Medicine, China Medical University, Taichung, Taiwan \\ ${ }^{2}$ Graduate Institute of Chinese Medical Science, China Medical University, Taichung, Taiwan \\ ${ }^{3}$ Management Office for Health Data, China Medical University Hospital, Taichung, Taiwan \\ ${ }^{4}$ College of Medicine, China Medical University, Taichung, Taiwan \\ ${ }^{5}$ Department of Chinese Medicine, China Medical University Hospital, Taichung, Taiwan \\ ${ }^{6}$ Research Center for Chinese Medicine \& Acupuncture, China Medical University, Taichung, Taiwan \\ ${ }^{7}$ Department of Healthcare Administration, College of Health Science, Asia University, Taichung, Taiwan \\ ${ }^{8}$ Department of Public Health, College of Public Health, China Medical University, Taichung, Taiwan \\ Correspondence should be addressed to Tsai-Chung Li; tcli@mail.cmu.edu.tw
}

Received 22 May 2016; Revised 15 August 2016; Accepted 14 September 2016

Academic Editor: Raffaele Capasso

Copyright ( 2016 Yu-Jun Wang et al. This is an open access article distributed under the Creative Commons Attribution License, which permits unrestricted use, distribution, and reproduction in any medium, provided the original work is properly cited.

\begin{abstract}
Leukemia is the most common malignancy among all childhood cancers and is associated with a low survival rate in adult patients. Since 1995, the National Health Insurance (NHI) program in Taiwan has been offering insurance coverage for Traditional Chinese Medicine (TCM), along with conventional Western medicine (WM). This study analyzes the status of TCM utilization in Taiwan, in both pediatric and adult patients with leukemia. A retrospective cohort study was conducted using population-based National Health Insurance Research Database of Registry of Catastrophic Illness, involving patient data from 2001 to 2010 and follow-up data through 2011. The effectiveness of TCM use was evaluated. Relevant sociodemographic data showed that both pediatric and adult patients who were TCM users one year prior to leukemia diagnosis were more likely to utilize TCM services for cancer therapy. A greater part of medical expenditure of TCM users was lower than that of TCM nonusers, except little discrepancy in drug fee of adult patients. The survival rate is also higher in TCM users. Altogether, these data show that TCM has the potential to serve as an adjuvant therapy when combined with conventional WM in the treatment of patients with leukemia.
\end{abstract}

\section{Introduction}

Leukemia originates in the bone marrow and results in the production of a large number of abnormal white blood cells. The four main subtypes of leukemia are acute lymphoblastic leukemia (ALL), acute myelogenous leukemia (AML), chronic lymphocytic leukemia (CLL), and chronic myelogenous leukemia (CML) [1]. Leukemia is the most common malignancy diagnosed in children accounting for greater than $30 \%$ of all childhood cancers [2]. While ALL is the most prevalent cancer in childhood and also the first leading cause of death from cancer within 20 years of age [3], AML is more common in older adults [4]. Patients with leukemia may undergo chemotherapy or hematopoietic stem cell transplants, which are associated with massive physical suffering and economic burden $[5,6]$.

The National Health Insurance (NHI) program has offered insurance cover for Traditional Chinese Medicine (TCM) and conventional Western medicine (WM) since 1995. Several studies have demonstrated that TCM as adjuvant therapy can help improve the quality of life and alleviate the side effects associated with WM use. Importantly, it can enhance the efficacy of WM treatment against tumors and improve the survival rate [7-13]. For instance, one study indicated that TCM combined with chemotherapy significantly elevates survival rate and improves the quality of 
life in patients with advanced non-small-cell lung carcinoma [12]. Another observational study demonstrated that TCM as adjuvant therapy helps to lower the risk of mortality relative to "TCM nonusers" in advanced breast cancer [9]. Apart from case reports and standardized questionnaire surveys, there are few large-scale studies on TCM use among leukemia patients $[14,15]$. Thus, our study aimed to explore the determinants of TCM utilization in both pediatric and adult leukemia patients and further evaluate the overall survival of patients.

\section{Materials and Methods}

2.1. Research Data Sources. The NHI program, initiated in 1995 , currently covers nearly $99.6 \%$ of the residents in Taiwan. The Bureau of National Health Insurance has contracts and cooperative agreements with nearly $97 \%$ of all hospitals and $92 \%$ of all clinics. This study included National Health Insurance Research Database (NHIRD) claim datasets that are safe from counterfeits due to the severe penalties involved [16]. The NHIRD datasets used in this study consisted of registry for beneficiaries, ambulatory and inpatient care claims, and registry for catastrophic illness from 1999 to 2011. Besides these, the NHIRD database contains demographic data, dates of visits, International Classification of Diseases, Ninth Revision, Clinical Modification (ICD-9-CM) diagnostic codes, complete prescription details, and expenditure incurred by the beneficiaries. Data for detailed diagnoses and treatments provided by physicians were included. On the other hand, all cancer cases registered in the catastrophic illness database were eligible for an exemption from copayment. Our study was approved by the Joint Institutional Review Board of Public Health, Social and Behavioral Science Committee, China Medical University and Hospital.

2.2. Study Population. Our population-based retrospective cohort study included newly diagnosed patients with leukemia (ICD-9-CM code 204-208) identified from the registry of catastrophic illness between January 1, 2001, and December 31, 2010. In Taiwan health care system, the diagnosis of leukemia cases was done by WM physicians and cannot be done by TCM physicians because all diagnosis tests, including regular blood routine test (CBC/DC), blood smear (observing blood cell morphology), bone marrow biopsy (observing bone marrow cell morphology), and symptoms and signs, can be performed only by WM physicians. The index date was the initial date of leukemia diagnosis and all patients were followed up through December 31, 2011, or until the patients' death date within this interval or withdrawal from NHI. The index date was the date of first diagnosis. We analyzed 2,355 newly diagnosed pediatric patients ( $0-18$ years old) and 10,208 newly diagnosed adult patients (19-80 years old) with leukemia. Patients who visited TCM physicians and used TCM at least once after being diagnosed with leukemia were deemed "TCM users" and the rest were deemed "TCM nonusers."

2.3. Comorbidities and Classification of Expenditures. This study considered TCM and WM ambulatory care, including
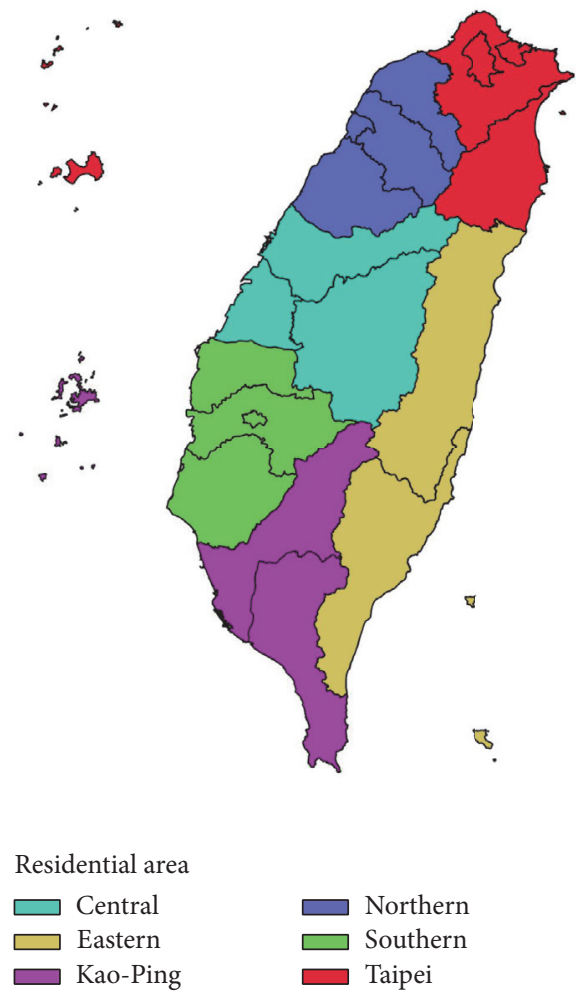

FIGURE 1: The residential areas of the study population.

dates of visit, date of birth, patient gender, medical facility and department visited, prescribing physician, dispensing pharmacist, three items from the ICD-9-CM codes, primary procedure, type of copayment, and paid amounts. We also analyzed the coexisting diseases in the leukemia patients based on the ICD-9-CM codes. Types of expenditure included fees for consultation, treatment and medical supplies, diagnosis fee, and drug fee.

2.4. Sociodemographic Factors and Urbanization Levels of Residential Area. The sociodemographic factors included age, gender, insurance premium amount, and the insured unit. Pediatric patients were further divided into 3 subgroups: 0-6, 7-12, and 13-18 years. Similarly, the adult patients were also divided into 3 subgroups: 19-40, 4160 , and $61-80$ years. The amount of insurance premium, determined from the individual working salary, was classified into four levels: $<20,000,20,000-39,999,40,000-59,999$, and $>60,000 \mathrm{NT} \$ /$ month. The residential areas of the study population comprised 6 areas: Northern area, Taipei, Central area, Southern area, Eastern area, and Kao-Ping area (Figure 1). Furthermore, the urbanization level of the townships in Taiwan was categorized according to educational level of the population, population density, the ratio of elder people, and occupation in general [17]. The insured unit included government, school, private enterprise, occupational member, farmer and fishermen, low-income household, and veterans.

2.5. Statistical Analysis. All analyses were performed separately for the pediatric and adult patient groups. The 
continuous variables were evaluated using means, standard deviations, and 95\% confidence interval (CI), whereas categorical variables were evaluated using the numbers, percentages, and 95\% CI. To compare the differences in continuous variables between TCM users and nonusers, Student's $t$ test was used, whereas Chi-square test was used to analyze the categorical variables. Furthermore, the adjusted odds ratio (OR), calculated using a multivariate logistic regression analysis, was used to explore the determinants of TCM utilization. The Kaplan-Meier estimator with a log-rank test was applied to evaluate the effect of TCM use on overall survival. Pairwise comparisons of overall survival among groups of no, low, and high TCM use were adjusted by Bonferroni correction. All $p$ values were calculated using two-sided tests, and the threshold for statistical significance was set at $p<0.05$. All analyses were performed using Statistical Analysis System (SAS) version 9.3 (SAS Institute Inc., Cary, NC, USA).

\section{Results}

3.1. Factors Associated with TCM Use. A total of 292 (12.40\%) pediatric patients and $936(9.17 \%)$ adult patients availed TCM outpatient services. In these leukemia patients, we observed that patients who were TCM users one year prior to the leukemia diagnosis and who resided in central area were more likely to utilize TCM services (Table 1). The mean numbers of days taking TCM in pediatric and adult leukemia patients were $184(\mathrm{SD}=328)$ days and $107(\mathrm{SD}=254)$ days, respectively. The mean outpatient department (OPD) visits for TCM in pediatric and adult leukemia patients were $20.3(\mathrm{SD}=35.3)$ and $10.5(\mathrm{SD}=22.8)$, respectively. The distributions of cancer type were similar between TCM users and nonusers in both pediatric and adult leukemia patients.

3.2. Medical Institutes. TCM users within pediatric leukemia patients engaged private hospitals more often for outpatient services than TCM pediatric nonusers $(42.15 \%$ versus $38.70 \%)$. Similar trend was also seen within adult leukemia patients $(36.17 \%$ versus $34.20 \%)$. On the contrary, private clinics were more often engaged for outpatient services by TCM nonusers of pediatric patients when compared to TCM users (41.37\% versus $35.22 \%$ ) as well as by TCM adult nonusers (43.32\% versus $41.13 \%$ ) (Table 2 ).

3.3. Coexisting Diseases. We observed that lymphoid leukemia and myeloid leukemia were the two major diagnoses in these leukemia patients. Lymphoid leukemia was common in children and myeloid leukemia was diagnosed mainly in adults. Acute upper and lower respiratory infections were commonly diagnosed in both pediatric and adult patients, irrespective of TCM use by the patients (Table 3).

3.4. Expenditures. According to our analysis, drug fee was the major component of all outpatient medical expenditures (Table 4). For both pediatric and adult leukemia patients, the average cost of total amount per visit was higher for TCM users than that of TCM nonusers. In adult leukemia patients, the average cost of drug fee per visit in TCM users was higher than that of TCM nonusers. Nevertheless, the differences in average costs between these two groups were indeed minimal (2,557.98 NT\$ versus 2,535.07 NT\$). The costs of other items per visit in TCM users in adult leukemia patients were significantly lower than those of TCM nonusers, including fees for consultation, treatment, medical supply, and diagnosis fee. In pediatric patients, the average cost per visit in TCM users was consistently higher than that of TCM nonusers for all items including fees for consultation, treatment, medical supply, diagnosis fee, and drug fee.

\subsection{Most Commonly Prescribed TCM Single and Formula} Products. Details of the TCM single and formula products most frequently prescribed by TCM physicians are shown in Table 5. In pediatric leukemia patients, Radix Astragali membranaceus was the most commonly prescribed TCM single product, followed by Bulbus Fritillariae thunbergii, and Herba Hedyotis diffusa. Moreover, Xiang Sha Liu Jun $\mathrm{Zi}$ Tang was the most frequently prescribed TCM formula product, followed by Yu Ping Feng San and Zuo Gui Wan. The top three most commonly used TCM single products for adult leukemia patients were as follows, from the most common to the least: Radix Astragali membranaceus, Radix et Rhizoma Salviae miltiorrhizae, and Fructus Ligustri Lucidi. Moreover, the top three most commonly prescribed TCM single products were Gui Pi Tang, Liu Wei Di Huang Wan, and Zuo Gui Wan.

3.6. Overall Survival. We found that the overall survival rate was higher in TCM users compared to TCM nonusers in both pediatric and adult patients with leukemia (both $p<$ 0.001) (Figure 2). During the 10 years that we followed, both pediatric and adult patients with leukemia who were TCM users had near $10 \%$ higher survival probability in comparison to the corresponding group of TCM nonusers patients. To further examine whether the overall survival rates were influenced by the number of days taking TCM and number of OPD visits for TCM use, we categorized these two variables using their median values as cutoff points (Figures 3 and 4). We observed that the overall survival rates were significantly different in subgroups of number of days taking TCM and OPD visits for TCM use in both pediatric and adult patients with leukemia (all $p<0.001$ ). We observed a dose-response relationship among three subgroups of TCM use according to either patients with number of days taking TCM or number of OPD visits for TCM use. The highest overall survival rates were found in patients with high number of days taking TCM ( $\geq 42.5$ for pediatric patients and $\geq 28$ for adult patients), which were significantly higher than those with low number of days taking TCM $(<42.5$ for pediatric patients and $<28$ for adult patients) $(p=0.0049$ for pediatric patients and $p<0.0001$ for adult patients) and were also significantly higher than those who were TCM nonusers (both $p<$ 0.001 for pediatric and adult patients). The differences in overall survival curves were different between patients with low number of days taking TCM and TCM nonusers ( $p=$ 0.0051 and $<0.001$ for pediatric and adult patients, resp.). Similarly, we observed significant pairwise differences in 


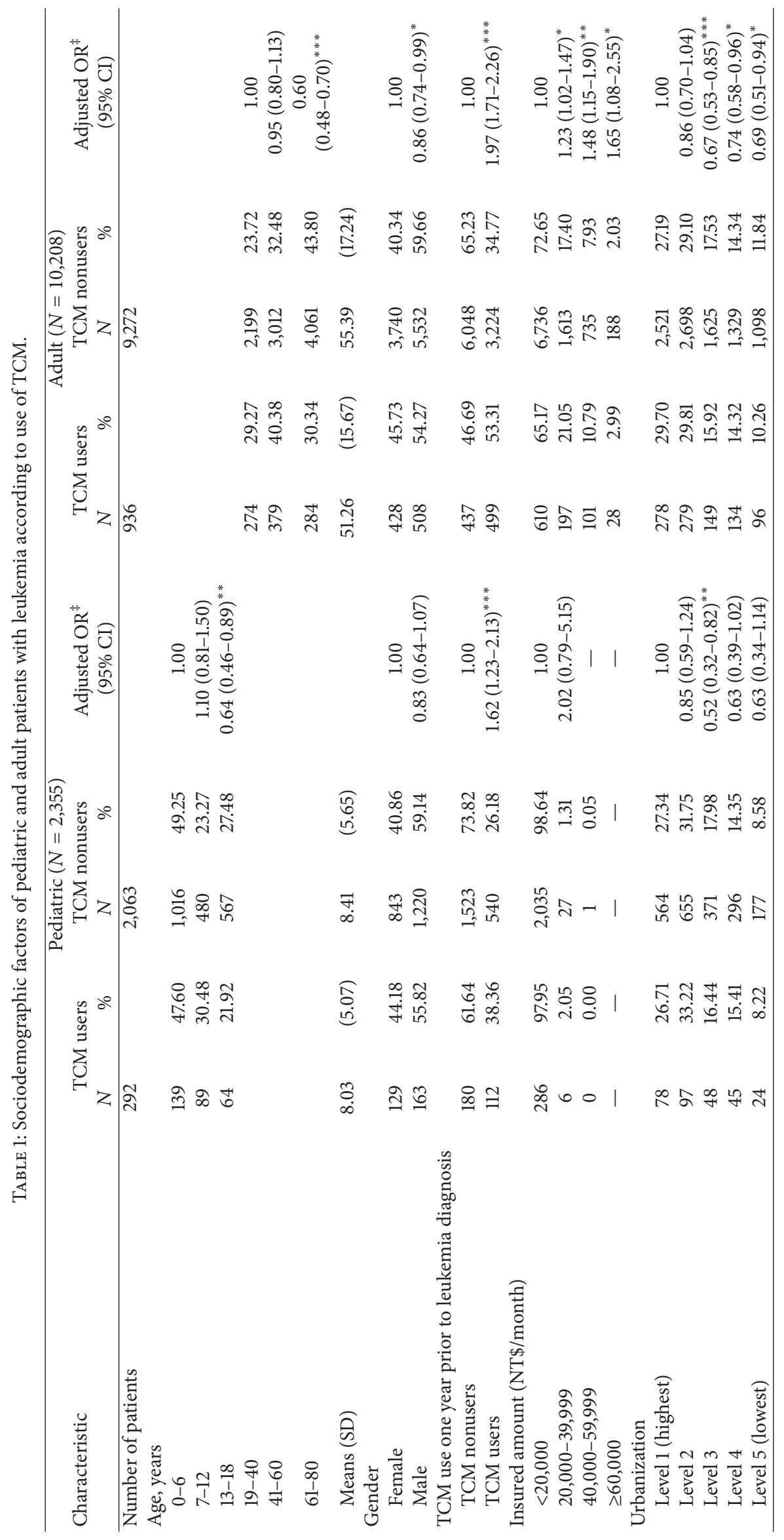




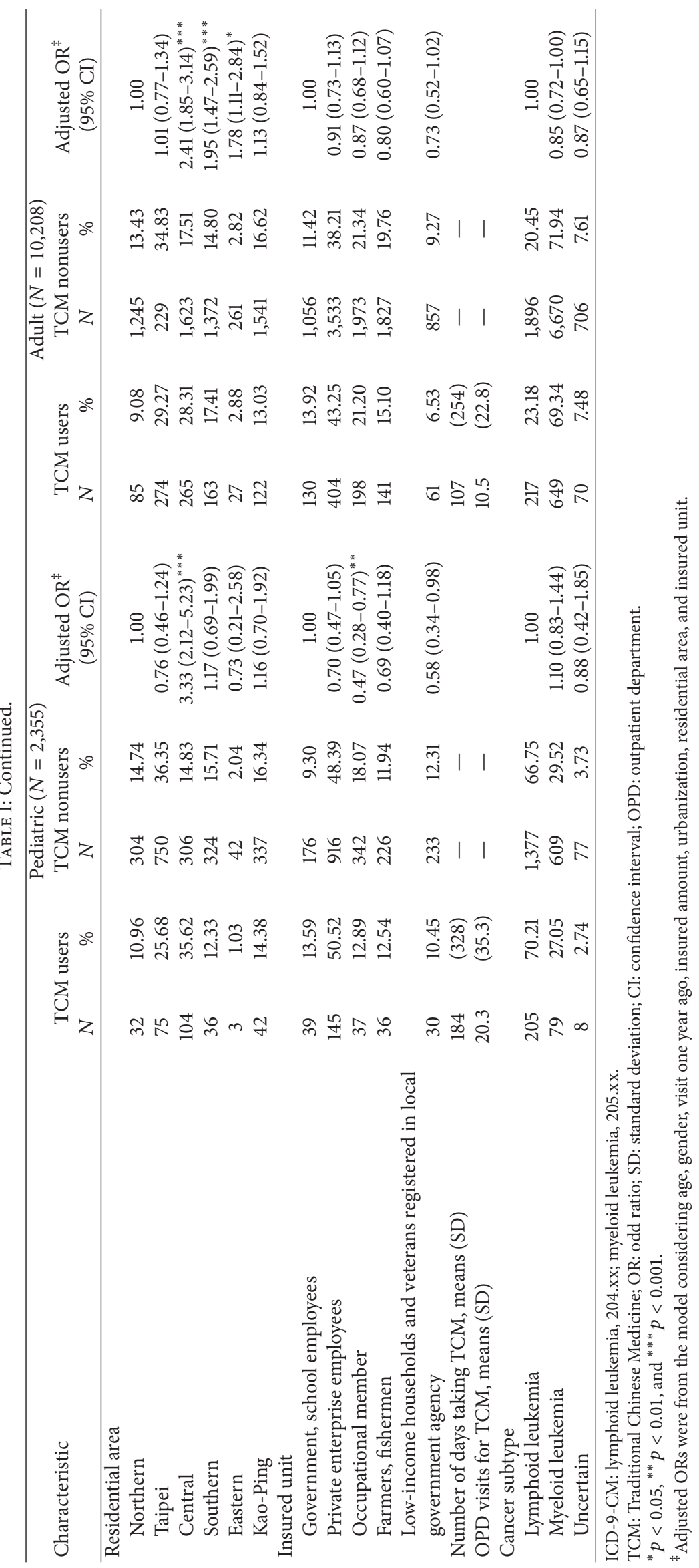


TABLE 2: Distributions of outpatient service providers for pediatric and adult patients with leukemia during 2001-2011.

\begin{tabular}{|c|c|c|c|c|c|}
\hline \multirow{2}{*}{ Outpatient services providers } & \multicolumn{2}{|c|}{ TCM users } & \multicolumn{2}{|c|}{ TCM nonusers } & \multirow{2}{*}{$p$ value for $\chi^{2}$} \\
\hline & Visits & Percentage (95\% CI) & Visits & Percentage $(95 \% \mathrm{CI})$ & \\
\hline \multicolumn{6}{|l|}{ Pediatric } \\
\hline Type of provider & & & & & $<0.001$ \\
\hline Public hospitals & 20,407 & $22.33(22.06,22.6)$ & 80,522 & $19.47(19.35,19.59)$ & \\
\hline Public Chinese medicine hospitals & 20 & $0.02(0.01,0.03)$ & 92 & $0.02(0.02,0.03)$ & \\
\hline Private hospitals & 38,522 & $42.15(41.83,42.47)$ & 160,049 & $38.70(38.55,38.85)$ & \\
\hline Private Chinese medicine hospitals & 86 & $0.09(0.07,0.11)$ & 191 & $0.05(0.04,0.05)$ & \\
\hline Public clinics & 160 & $0.18(0.15,0.2)$ & 1,497 & $0.36(0.34,0.38)$ & \\
\hline Private clinics & 32,187 & $35.22(34.91,35.53)$ & 171,108 & $41.37(41.22,41.52)$ & \\
\hline Other medicine service providers & 9 & $0.01(0,0.02)$ & 103 & $0.02(0.02,0.03)$ & \\
\hline Total & 91,391 & & 413,562 & & \\
\hline \multicolumn{6}{|l|}{ Adult } \\
\hline Type of provider & & & & & $<0.001$ \\
\hline Public hospitals & 52,167 & $20.64(20.49,20.80)$ & 354,202 & $19.77(19.71,19.83)$ & \\
\hline Public Chinese medicine hospitals & 400 & $0.16(0.14,0.17)$ & 905 & $0.05(0.05,0.05)$ & \\
\hline Private hospitals & 91,396 & $36.17(35.98,36.36)$ & 612,655 & $34.20(34.13,34.27)$ & \\
\hline Private Chinese medicine hospitals & 614 & $0.24(0.22,0.26)$ & 4,044 & $0.23(0.22,0.23)$ & \\
\hline Public clinics & 3,782 & $1.5(1.45,1.54)$ & 40,716 & $2.27(2.25,2.29)$ & \\
\hline Private clinics & 103,932 & $41.13(40.94,41.32)$ & 776,052 & $43.32(43.24,43.39)$ & \\
\hline Other medicine service providers & 399 & $0.16(0.14,0.17)$ & 3,026 & $0.17(0.16,0.17)$ & \\
\hline Total & 252,690 & & $1,791,600$ & & \\
\hline
\end{tabular}

TCM: Traditional Chinese Medicine; CI: confidence interval.

overall survival function among three subgroups of TCM use based on number of OPD visits.

\section{Discussion}

This study investigated the potential of TCM use as an adjuvant therapy for leukemia patients, both pediatric and adult, in Taiwan undergoing treatment with WM. The determinants for TCM utilization for leukemia patients in Taiwan, such as medical expenditure, and therapeutic effects were only scarcely reported in the past [18]. Our study shows that the combination therapy using TCM and WM for patients with leukemia does not cause additional financial burden relative to using WM only. Importantly, TCM use is associated with longer survival.

We observed that TCM utilization was more prevalent in pediatric leukemia patients rather than adults. We assume that children can barely tolerate the extremely uncomfortable symptoms and feelings accompanying chemotherapy, as previously suggested [19]. Studies show that TCM can alleviate adverse effects of WM and improve quality of life in cancer patients $[7,10,11,13]$. Andersen's health behavior model, which is advocated by many scholars of sociology and public health, claims that the use of health services is determined by predisposing factors, enabling factors, and need factors [20]. In our study, significant factors for TCM use included predisposing factors of gender and health beliefs, such as TCM use one year prior to leukemia diagnosis. Enabling factors observed were height of medical insurance, residential area, and urbanization level, which were similar to those reported by Liao et al. $[21,22]$. On the other hand, a particularly higher prevalence of TCM utilization was observed in central Taiwan, which has the highest per capita ratio of TCM physicians.

Radix Astragali membranaceus and Fructus Ligustri Lucidi were in the top 3 herbs for adult users and Radix Astragali membranaceus was the top one herbs for pediatric users. Both of these herbs have a function that increases white blood cell count [23]. Due to the indication of leukemia being a consistently elevated white blood cell count, some physicians thought using the herbs that increase white blood cell count is not recommended because of the potential exacerbation of leukemia. Given the findings of the present study, these herbs may increase healthy WBC without aggravating the leukemia. This can be supported by the prior studies of these two herbs demonstrating that they bolster host immune response for cytotoxic activity by promoting apoptosis of tumor cells and may help the recovery from chemotherapy or radiation treatments [18, 24-27]. The findings of the present study provide important information for TCM physicians and patients with leukemia.

The National Health Insurance program was started on March 1, 1995. In 2011, 22.60 million out of 22.96 million Taiwan residents were enrolled in this program. The comprehensive NHI database is considered an appropriate source to assess certain diseases. In addition, the NHI database provides abundant sample population size and details in studies, eliminating the bias associated with a limited sample size.

Our study has several limitations, though. Firstly, some patients visiting TCM physicians preferred to receive decoction formulations of Chinese medicine, which were self-paid 


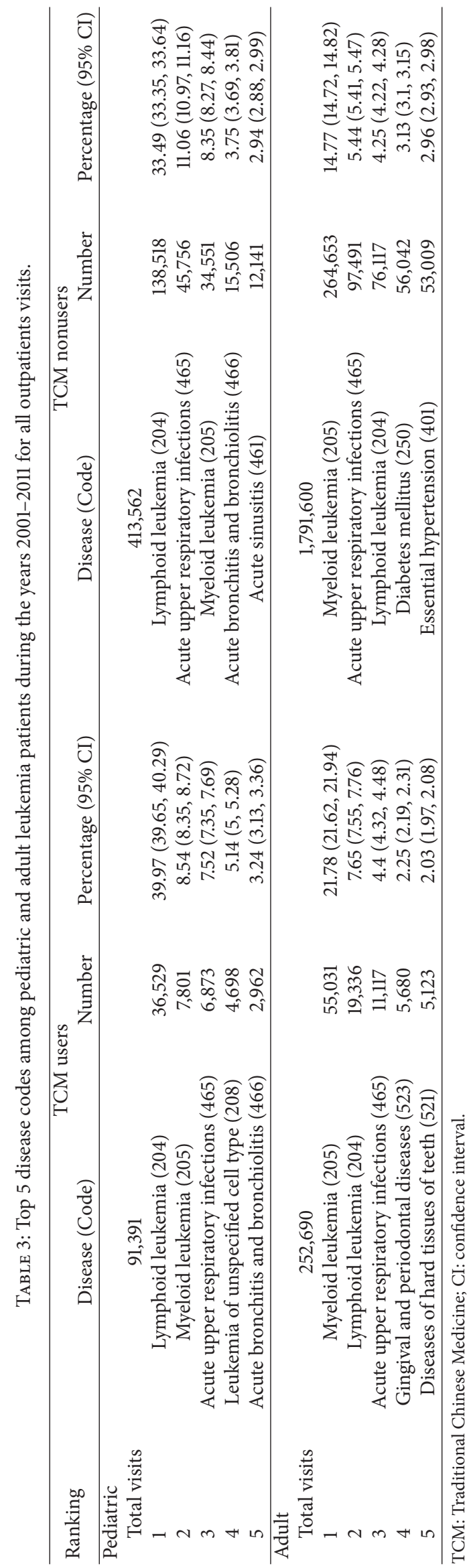




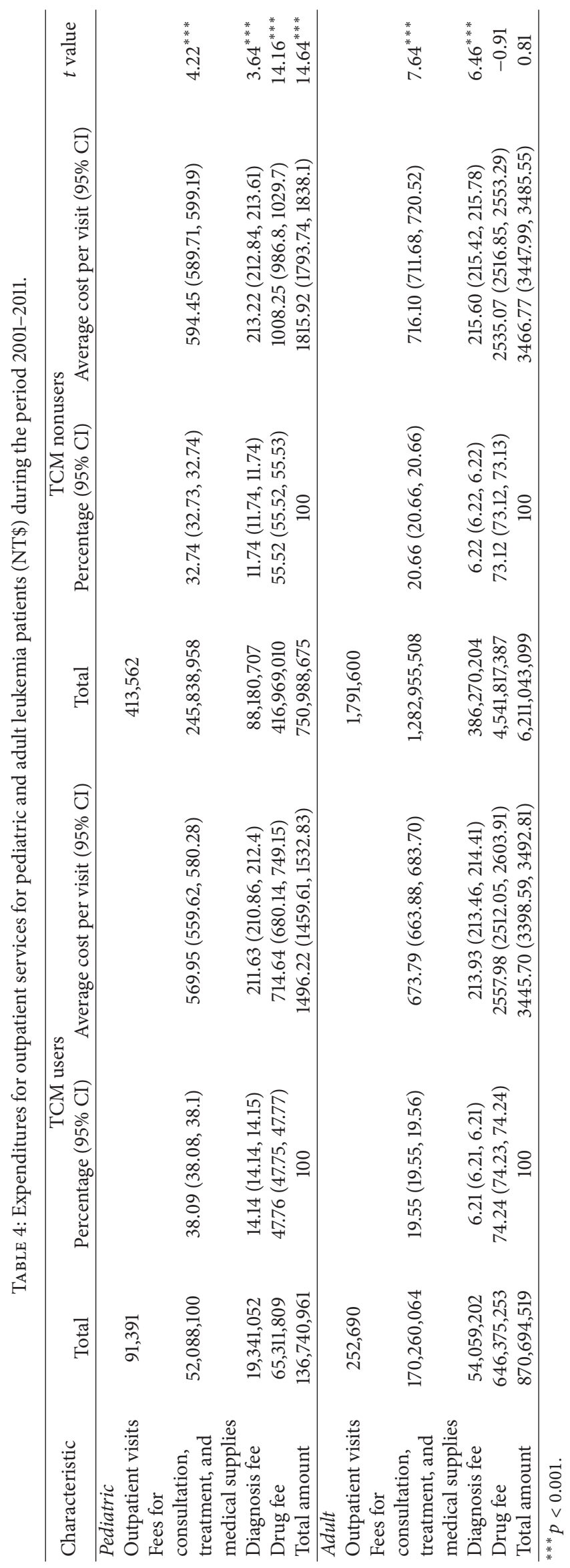




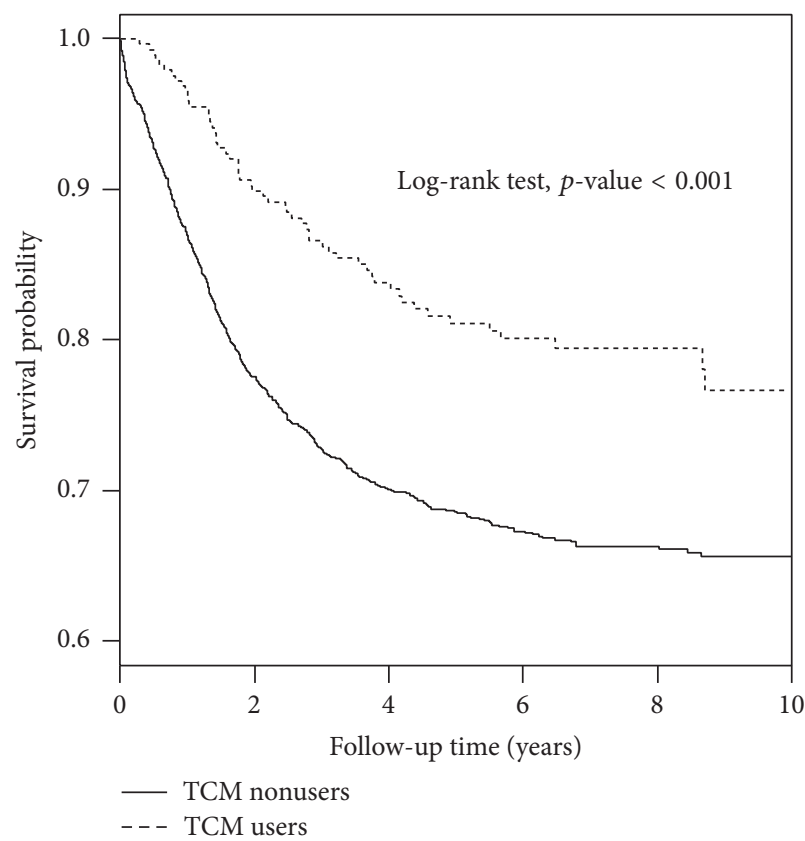

(a) Pediatric

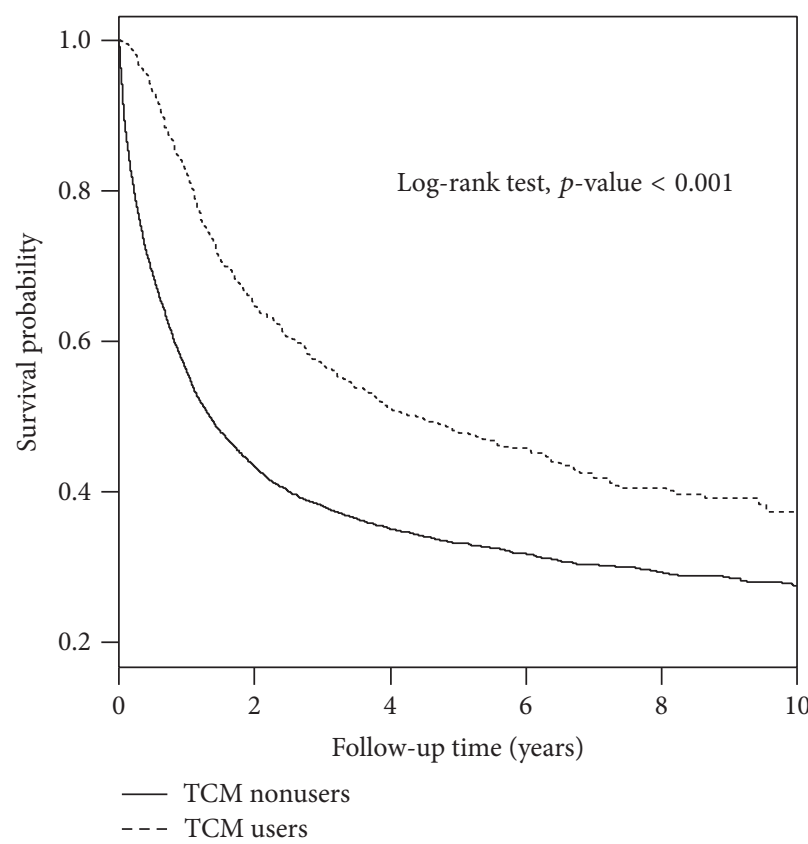

(b) Adult

FIGURE 2: Kaplan-Meier curves of overall survival in patients with leukemia based on TCM use during the follow-up period.

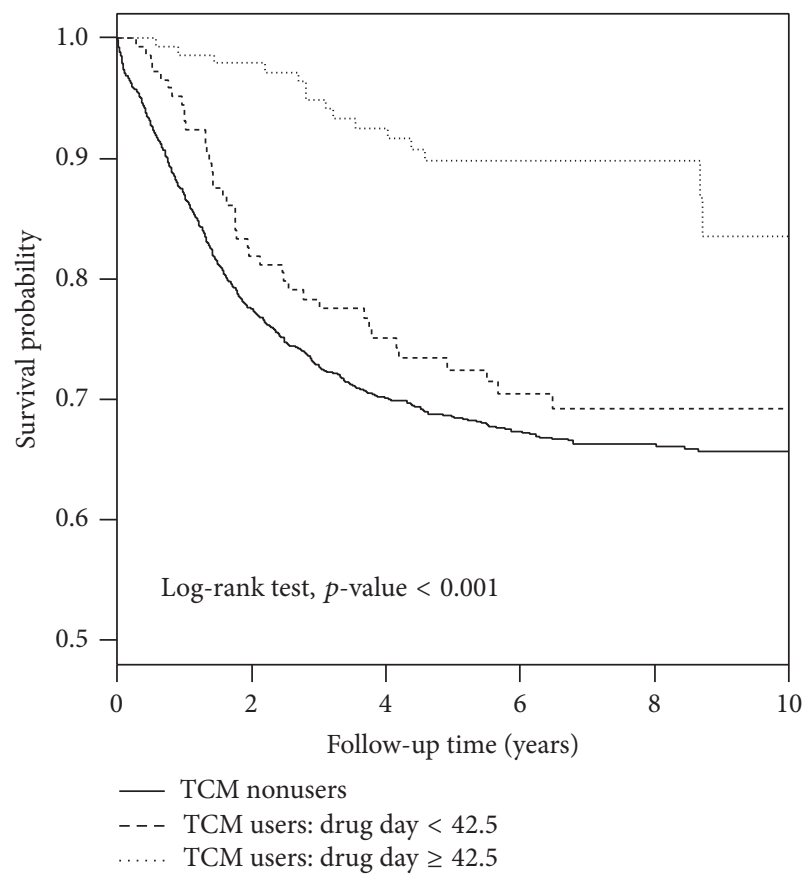

(a) Pediatric

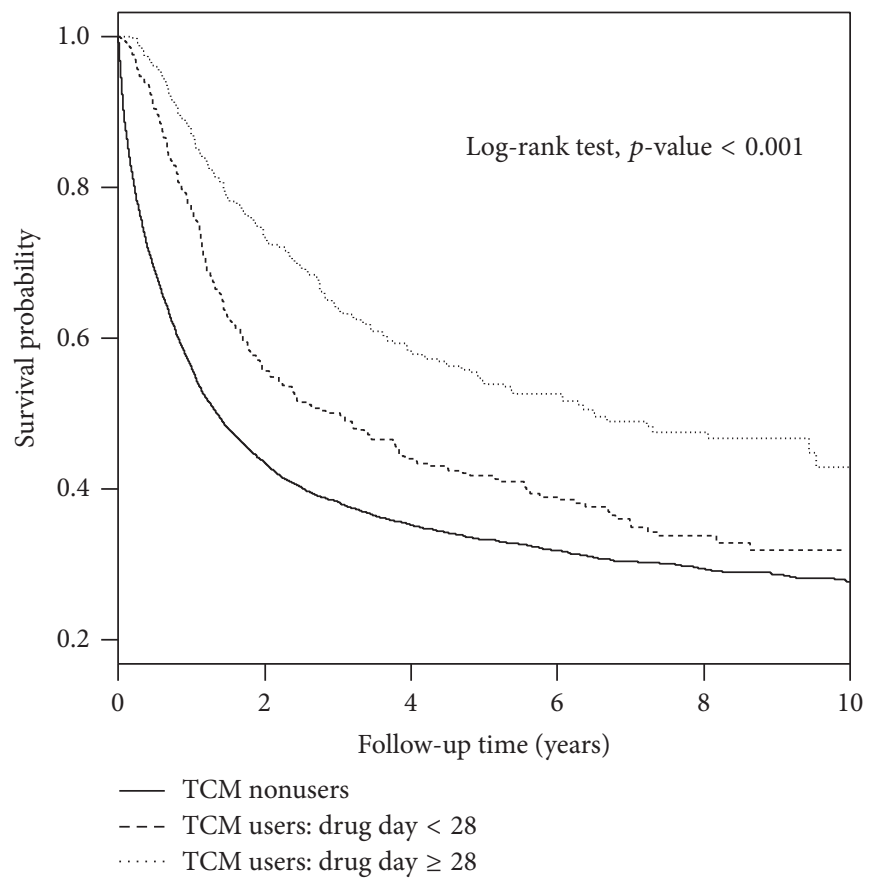

(b) Adult

Figure 3: Kaplan-Meier curves of overall survival in patients with leukemia based on number of drug days for TCM use during the follow-up period. (a) TCM nonusers versus TCM user: days taking TCM $<42.5, p=0.0051$. TCM nonusers versus TCM user: days taking TCM $\geq 42.5$, $p<0.001$. TCM user: days taking TCM $<42.5$ versus TCM user: days taking TCM $\geq 42.5, p=0.0049$. (b) TCM nonusers versus TCM user: days taking TCM $<28, p<0.001$. TCM nonusers versus TCM user: days taking TCM, $p<0.001$. TCM user: days taking TCM $<28$ versus TCM user: days taking TCM $\geq 28, p=0.0001$. 
TABLE 5: Top ten formula CHPs and single CHPs prescribed by TCM physicians to treat pediatric and adult patients with leukemia in Taiwan, 2000-2011.

\begin{tabular}{|c|c|c|c|c|}
\hline & \multicolumn{2}{|l|}{ Single CHPs } & \multicolumn{2}{|c|}{ Formula CHPs } \\
\hline & Name & Number, frequency (\%) & Name & Number, frequency (\%) \\
\hline \multirow{10}{*}{ Pediatric } & Radix Astragali membranaceus & $103(5.80)$ & Xiang Sha Liu Jun Zi Tang & $36(7.86)$ \\
\hline & Bulbus Fritillariae thunbergii & $87(4.90)$ & Yu Ping Feng San & $27(5.90)$ \\
\hline & Herba Hedyotis diffusa & $74(4.16)$ & Zuo Gui Wan & $25(5.46)$ \\
\hline & Radix Scutellariae baicalensis & $65(3.66)$ & Bu Zhong Yi Qi Tang & $24(5.24)$ \\
\hline & Radix Puerariae lobatae & $57(3.21)$ & Shen Ling Bai Zhu San & $20(4.37)$ \\
\hline & Radix Glycyrrhizae uralensis & $52(2.93)$ & Bao He Wan & $18(3.93)$ \\
\hline & Herba Houttuyniae cordata & $45(2.53)$ & Xin Yi San & $16(3.49)$ \\
\hline & Semen Ziziphi Spinosi & $34(1.91)$ & Ma Zi Ren Wan & $16(3.49)$ \\
\hline & Rhizoma Polygonati odorati & $32(1.80)$ & Liu Wei Di Huang Wan & $14(3.06)$ \\
\hline & Herba Scutellariae barbatae & $29(1.63)$ & Ge Gen Tang & $14(3.06)$ \\
\hline \multirow{10}{*}{ Adult } & Radix Astragali membranaceus & $173(4.07)$ & Gui Pi Tang & $83(6.18)$ \\
\hline & Radix et Rhizoma Salviae miltiorrhizae & $133(3.13)$ & Liu Wei Di Huang Wan & $53(3.95)$ \\
\hline & Fructus Ligustri Lucidi & $108(2.54)$ & Zuo Gui Wan & $39(2.91)$ \\
\hline & Sargentodoxa Cuneata & $86(2.02)$ & Gui Lu Er Xian Jiao & $39(2.91)$ \\
\hline & Herba Hedyotis diffusa & $82(1.93)$ & Sheng Mai San & $36(2.68)$ \\
\hline & Rhizoma Dioscoreae polystachya & $71(1.67)$ & Jia Wei Xiao Yao San & $35(2.61)$ \\
\hline & Bulbus Fritillaria thunbergii & $69(1.62)$ & Xue Fu Zhu Yu Tang & $27(2.01)$ \\
\hline & Cortex Lycii Radicis & $63(1.48)$ & Xiang Sha Liu Jun Zi Tang & $26(1.94)$ \\
\hline & Radix Glycyrrhizae uralensis & $59(1.39)$ & Xin Yi Qing Fei Tang & $25(1.86)$ \\
\hline & Semen Ziziphi Spinosae & $58(1.36)$ & Ren Shen Yang Rong Tang & $24(1.79)$ \\
\hline
\end{tabular}

TCM: Traditional Chinese Medicine; CHPs: Chinese herb products.

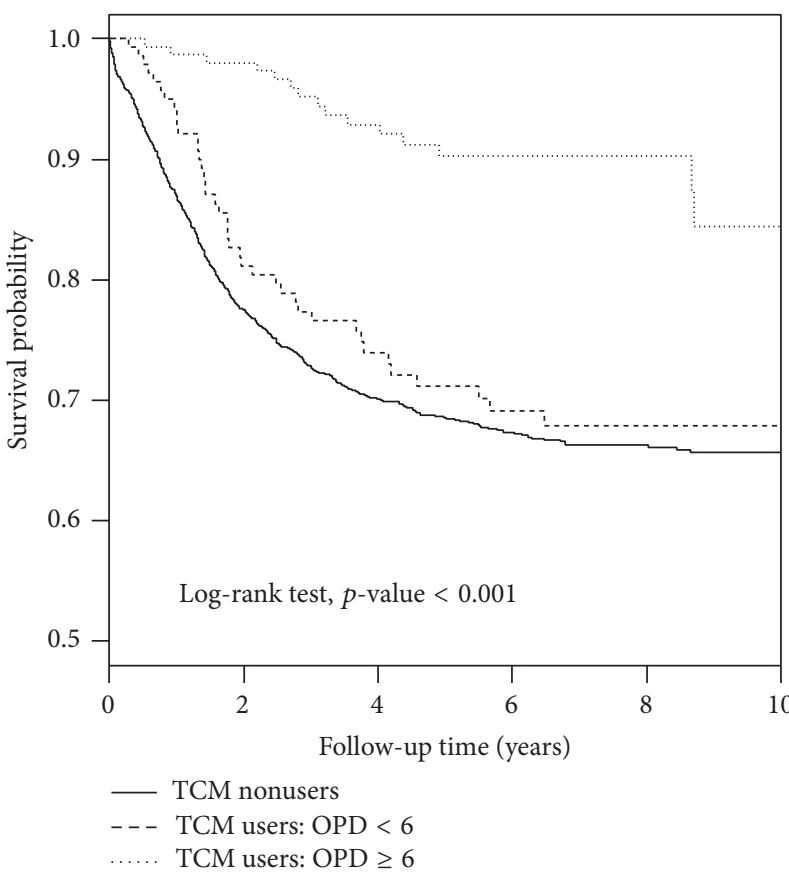

(a) Pediatric

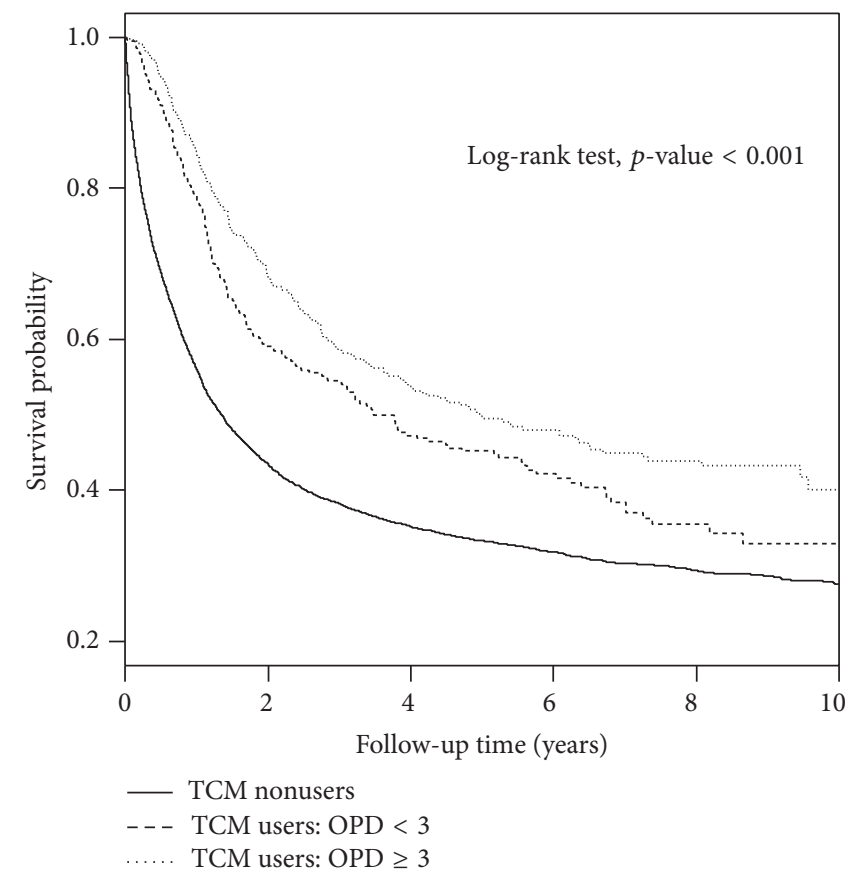

(b) Adult

FIGURE 4: Kaplan-Meier curves of overall survival in patients with leukemia based on number of outpatient department (OPD) visits for TCM use during the follow-up period. (a) TCM nonusers versus TCM user: OPD $<6, p=0.0075$; TCM nonusers versus TCM user: OPD $\geq 6, p<0.001$; TCM user: OPD $<42.5$ versus TCM user: OPD $\geq 6, p=0.0009$. (b) TCM nonusers versus TCM user: OPD $<3, p<0.001$; TCM nonusers versus TCM user: $\mathrm{OPD} \geq 3, p<0.001$; TCM user: $\mathrm{OPD}<3$ versus TCM user: $\mathrm{OPD} \geq 3, p<0.001$. 
and not covered by the NHI program. In addition, about $10 \%$ of the TCM clinics were not contracted to the NHI because of the low reimbursement rates provided by the NHI. Therefore, the prevalence of TCM use and costs of outpatient visits could be underestimated. Secondly, we only explored the efficacy of general TCM utilization, including overall survival, but we did not consider the subtypes of leukemia and TCM prescriptions, which should be investigated in further studies. Last, the database does not contain information on daily activity, dietary habits, and body mass index, which may also be factors for TCM utilization and health care costs. Further studies considering the above information are therefore warranted.

\section{Conclusion}

Our study investigated the TCM utilization and prevalence among pediatric and adult patients with leukemia in Taiwan. It shows that TCM as adjuvant therapy combined with WM may indeed alleviate adverse symptoms, improve quality of life, and prolong overall survival, without incurring excess medical expenditure. This line of study in the future should aim at ruling out the possible confounders such as diet, lifestyle behaviors of exercise, and psychological status. The results of our study could be useful to health-policy decision makers as well as clinical practitioners while considering the integration of TCM with WM.

\section{Competing Interests}

The authors declare that there is no conflict of interests regarding the publication of this manuscript.

\section{Authors' Contributions}

Yu-Jun Wang and Chung-Chih Liao contributed equally to this work.

\section{Acknowledgments}

The Taiwan Ministry of Health and Welfare Clinical Trial and Research Center of Excellence (MOHW105TDU-B-212-133019), China Medical University Hospital, Academia Sinica Taiwan Biobank Stroke Biosignature Project (BM10501010037), NRPB Stroke Clinical Trial Consortium (MOST 105-2325-B-039-003), Tseng-Lien Lin Foundation, Taichung, Taiwan, Taiwan Brain Disease Foundation, Taipei, Taiwan, and Katsuzo and Kiyo Aoshima Memorial Funds, Japan, supported this study.

\section{References}

[1] A. Redaelli, J. M. Stephens, B. L. Laskin, C. L. Pashos, and M. F. Botteman, "The burden and outcomes associated with four leukemias: AML, ALL, CLL and CML," Expert Review of Anticancer Therapy, vol. 3, no. 3, pp. 311-329, 2003.

[2] M. Belson, B. Kingsley, and A. Holmes, "Risk factors for acute leukemia in children: a review," Environmental Health Perspectives, vol. 115, no. 1, pp. 138-145, 2007.
[3] S. P. Hunger and C. G. Mullighan, "Acute lymphoblastic leukemia in children," The New England Journal of Medicine, vol. 373, no. 16, pp. 1541-1552, 2015.

[4] A. M. Almeida and F. Ramos, "Acute myeloid leukemia in the older adults," Leukemia Research Reports, vol. 6, pp. 1-7, 2016.

[5] A. K. Burnett, N. H. Russell, R. K. Hills et al., "Optimization of chemotherapy for younger patients with acute myeloid leukemia: results of the medical research council AML15 trial," Journal of Clinical Oncology, vol. 31, no. 27, pp. 3360-3368, 2013.

[6] S. Frey, C. R. Blankart, and T. Stargardt, "Economic burden and quality-of-life effects of chronic lymphocytic leukemia: a systematic review of the literature," PharmacoEconomics, vol. 34, no. 5, pp. 479-498, 2016.

[7] S. Chen, A. Flower, A. Ritchie et al., "Oral Chinese herbal medicine (CHM) as an adjuvant treatment during chemotherapy for non-small cell lung cancer: a systematic review," Lung Cancer, vol. 68, no. 2, pp. 137-145, 2010.

[8] J. S. Jacobson, S. B. Workman, and F. Kronenberg, "Research on complementary/alternative medicine for patients with breast cancer: a review of the biomedical literature," Journal of Clinical Oncology, vol. 18, no. 3, pp. 668-683, 2000.

[9] Y.-W. Lee, T.-L. Chen, Y.-R. V. Shih et al., "Adjunctive traditional Chinese medicine therapy improves survival in patients with advanced breast cancer: a population-based study," Cancer, vol. 120, no. 9, pp. 1338-1344, 2014.

[10] S. G. Li, H. Y. Chen, C. S. Ou-Yang et al., "The efficacy of chinese herbal medicine as an adjunctive therapy for advanced nonsmall cell lung cancer: a systematic review and meta-analysis," PLoS ONE, vol. 8, no. 2, Article ID e57604, 2013.

[11] M. Paul, B. Davey, B. Senf et al., "Patients with advanced cancer and their usage of complementary and alternative medicine," Journal of Cancer Research and Clinical Oncology, vol. 139, no. 9, pp. 1515-1522, 2013.

[12] Z. Y. Xu, C. J. Jin, C. C. Zhou et al., "Treatment of advanced nonsmall-cell lung cancer with Chinese herbal medicine by stages combined with chemotherapy," Journal of Cancer Research and Clinical Oncology, vol. 137, no. 7, pp. 1117-1122, 2011.

[13] J. Yarney, A. Donkor, S. Y. Opoku et al., "Characteristics of users and implications for the use of complementary and alternative medicine in Ghanaian cancer patients undergoing radiotherapy and chemotherapy: A Cross- Sectional Study," BMC Complementary and Alternative Medicine, vol. 13, article 16, 2013.

[14] T. Elsner, R. Muecke, O. Micke et al., "Survey on the worldwide chronic myeloid leukemia advocates network regarding complementary and alternative medicine," Journal of Cancer Research and Clinical Oncology, vol. 139, no. 6, pp. 1025-1031, 2013.

[15] M. Hensel, M. Zoz, and A. D. Ho, "Complementary and alternative medicine in patients with chronic lymphocytic leukemia," Supportive Care in Cancer, vol. 17, no. 1, pp. 47-52, 2009.

[16] H.-F. Chen, C.-A. Ho, and C.-Y. Li, "Age and sex may significantly interact with diabetes on the risks of lower-extremity amputation and peripheral revascularization procedures: evidence from a cohort of a half-million diabetic patients," Diabetes Care, vol. 29, no. 11, pp. 2409-2414, 2006.

[17] C.-C. Lin, C.-I. Li, C.-Y. Hsiao et al., "Time trend analysis of the prevalence and incidence of diagnosed type 2 diabetes among adults in Taiwan from 2000 to 2007: a population-based study," BMC Public Health, vol. 13, no. 1, article 318, 2013. 
[18] L. H. Huang, Q. J. Yan, N. K. Kopparapu, Z. Q. Jiang, and Y. Sun, "Astragalus membranaceus lectin (AML) induces caspasedependent apoptosis in human leukemia cells," Cell Proliferation, vol. 45, no. 1, pp. 15-21, 2012.

[19] F. Naja, M. Alameddine, M. Abboud, D. Bustami, and R. Al Halaby, "Complementary and alternative medicine use among pediatric patients with leukemia: the case of Lebanon," Integrative Cancer Therapies, vol. 10, no. 1, pp. 38-46, 2011.

[20] R. M. Andersen, "Revisiting the behavioral model and access to medical care: does it matter?" Journal of Health and Social Behavior, vol. 36, no. 1, pp. 1-10, 1995.

[21] Y.-H. Liao, C.-C. Lin, T.-C. Li, and J.-G. Lin, "Utilization pattern of traditional Chinese medicine for liver cancer patients in Taiwan," BMC Complementary and Alternative Medicine, vol. 12, article 146, 2012.

[22] Y.-H. Liao, J.-G. Lin, C.-C. Lin, and T.-C. Li, "Distributions of usage and the costs of conventional medicine and traditional Chinese medicine for lung cancer patients in Taiwan," EvidenceBased Complementary and Alternative Medicine, vol. 2013, Article ID 984876, 11 pages, 2013.

[23] H. Yeung, Handbook of Chinese Herbs and Formulas, Institute of Chinese Medicine, Los Angeles, Calif, USA, 1985.

[24] B. Hu, Q. Du, S. Deng et al., "Ligustrum lucidum Ait. fruit extract induces apoptosis and cell senescence in human hepatocellular carcinoma cells through upregulation of $\mathrm{p} 21$," Oncology Reports, vol. 32, no. 3, pp. 1037-1042, 2014.

[25] Y.-L. Kuo, C.-H. Chen, T.-H. Chuang et al., "Gene expression profiling and pathway network analysis predicts a novel antitumor function for a botanical-derived drug, PG2," EvidenceBased Complementary and Alternative Medicine, vol. 2015, Article ID 917345, 15 pages, 2015.

[26] Z. Pang, Z.-Y. Zhou, W. Wang et al., "The advances in research on the pharmacological effects of Fructus Ligustri Lucidi," BioMed Research International, vol. 2015, Article ID 281873, 5 pages, 2015.

[27] P. Zhang, H. Li, D. Chen, J. Ni, Y. Kang, and S. Wang, "Oleanolic acid induces apoptosis in human leukemia cells through caspase activation and poly(ADP-ribose) polymerase cleavage," Acta Biochimica et Biophysica Sinica, vol. 39, no. 10, pp. 803-809, 2007. 


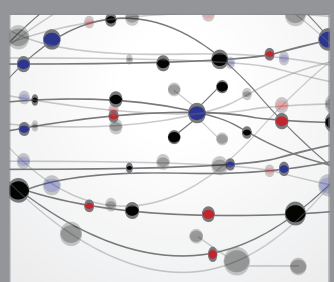

The Scientific World Journal
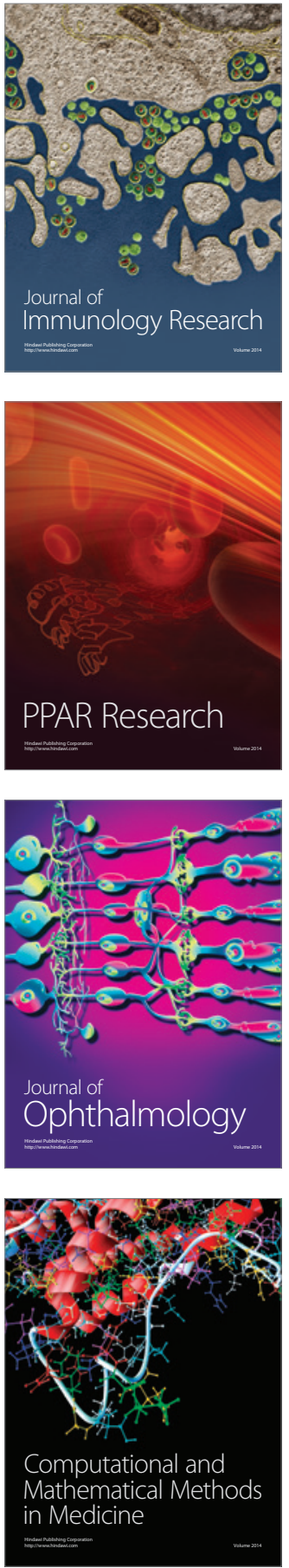

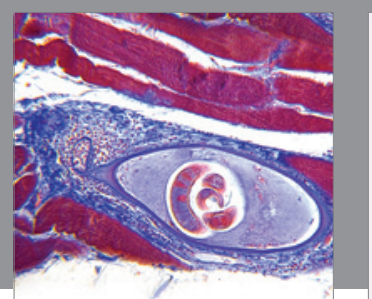

Gastroenterology Research and Practice

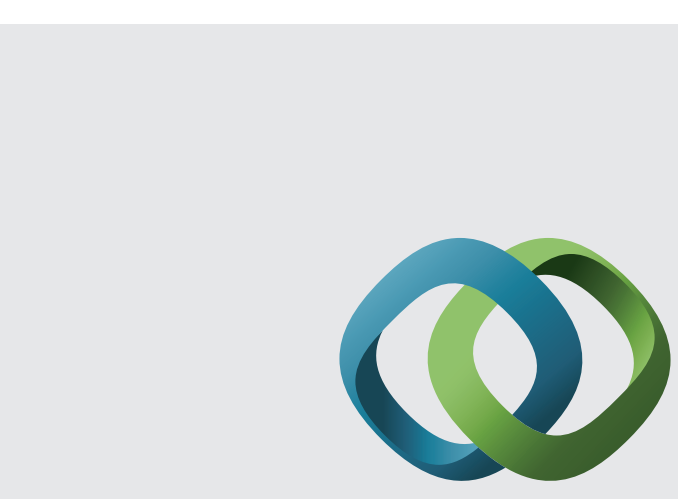

\section{Hindawi}

Submit your manuscripts at

http://www.hindawi.com
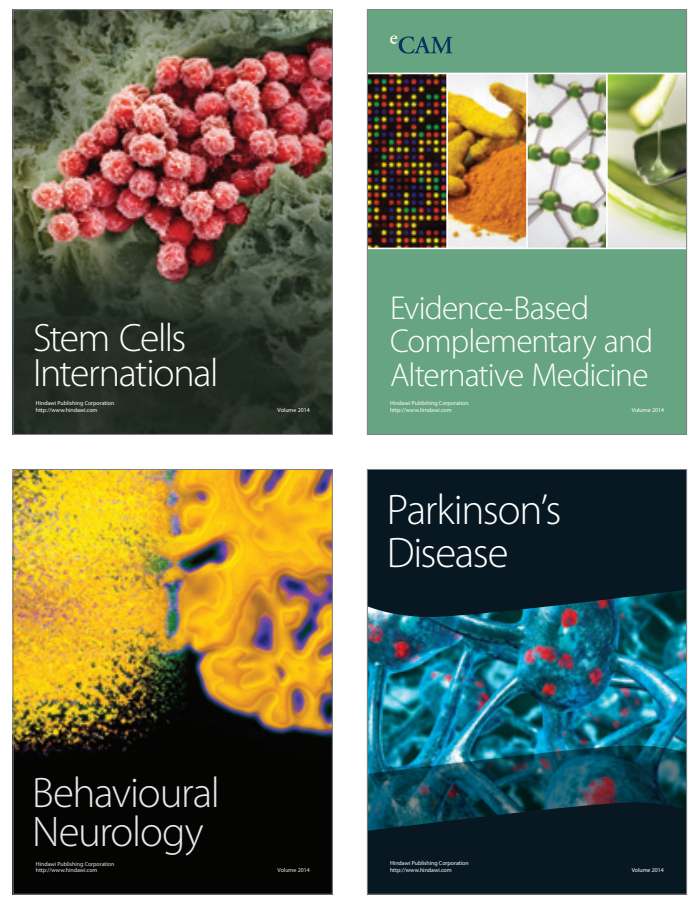
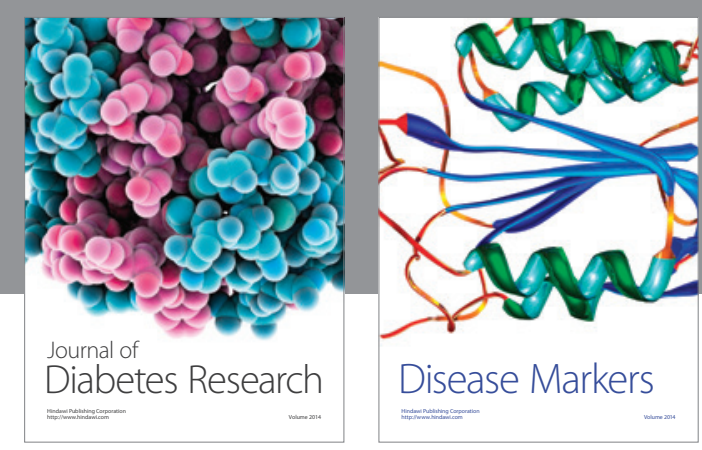

Disease Markers
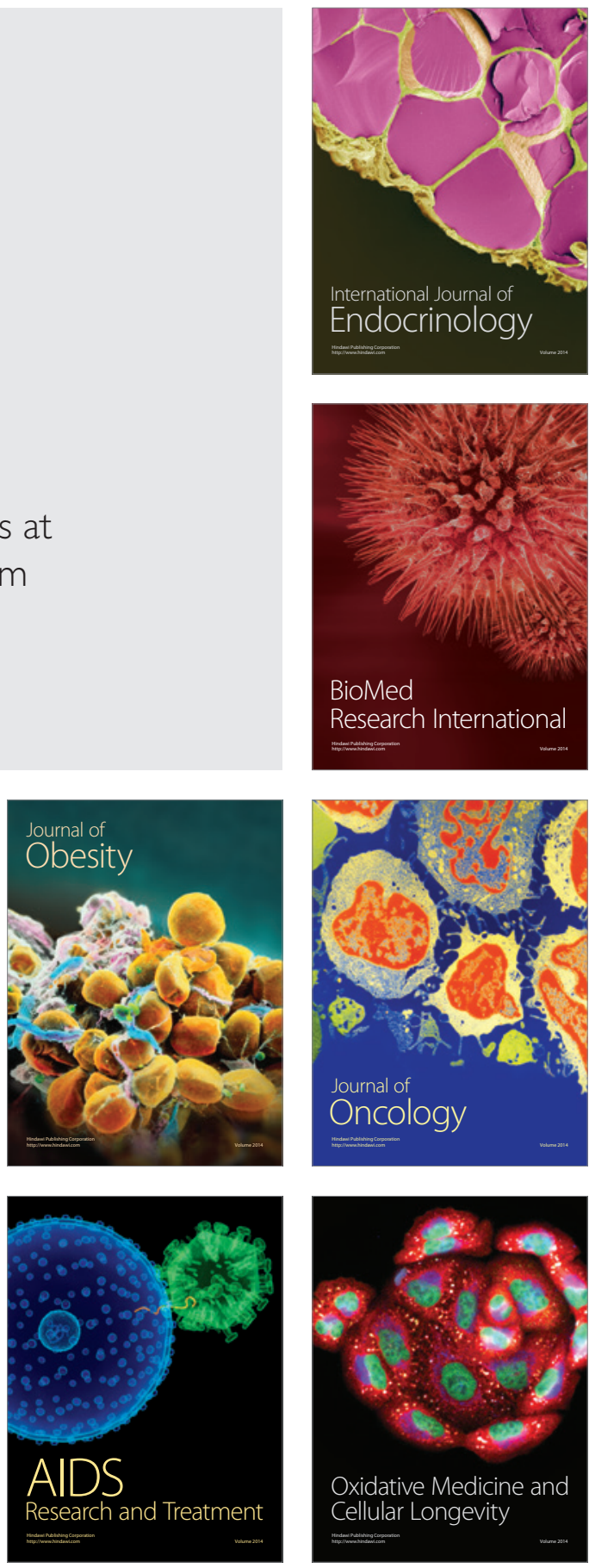